\title{
Predator-Prey Brain Storm Optimization for DC Brushless Motor
}

\author{
Haibin Duan ${ }^{1}$, Shuangtian $\mathrm{Li}^{1}$, and Yuhui $\mathrm{Shi}^{2}$ \\ ${ }^{1}$ State Key Laboratory of Virtual Reality Technology and Systems, School of Automation Science and Electrical Engineering, \\ Beihang University, Beijing 100191, China \\ ${ }^{2}$ Xi' an Jiaotong-Liverpool University, Suzhou, Jiangsu 215123, China
}

\begin{abstract}
Brain Storm Optimization (BSO) is a newly-developed swarm intelligence optimization algorithm inspired by a human being's behavior of brainstorming. In this paper, a novel predator-prey BSO model, which is named Predator-prey Brain Storm Optimization (PPBSO), is proposed to solve an optimization problem modeled for a DC brushless motor. The Predator-prey concept is adopted to better utilize the global information and improve the swarm diversity during the evolution process. The proposed algorithm is applied to solve the optimization problems in an electromagnetic field. The comparative results demonstrate that both PPBSO and BSO can succeed in optimizing design variables for a DC brushless motor to maximize its efficiency. Simulation results show PPBSO has better ability to jump out of local optima when compared with the original BSO. In addition, it demonstrates satisfactory stability in repeated experiments.
\end{abstract}

Index Terms-Brain storm optimization (BSO), brushless motor, electromagnetics, evolutionary computation, optimization.

\section{INTRODUCTION}

$\mathbf{S}$ WARM INTELLIGENCE (SI) optimization algorithms [1], including Genetic Algorithm (GA) [2]-[5], Particle Swarm Optimization (PSO) [6], Artificial Bee Colony (ABC) algorithm [7], and Biogeography-Based Optimization (BBO) [8], constitute an important part of evolutionary computation and have been widely used in the field of applied electromagnetics over the past decade. In contrast to conventional methods relying on gradient information, these algorithms operate on the basis of a searching swarm and interactions among its individuals. Each individual in the swarm searches within the searching space according to particular rules by taking the information of other peer individuals into account. As a result of such mechanisms, these algorithms exhibit good performance in solving nonlinear and discontinuous optimization problems.

A novel SI algorithm named Brain Storm Optimization (BSO) was first proposed by Shi in 2011 [9], which is inspired by the process of brainstorming, namely the mulling over of ideas by several individuals in an attempt to devise or find a solution to an optimization problem. For the optimization problem, each position in the searching space can be regarded as an idea. In each generation of the evolution, the ideas are gathered into different groups by a clustering operation, superior ones being cluster centers of each group. The ideas are then updated by searching in the neighborhood and combination with ideas from other clusters.

Different from other SI algorithms, which are inspired by the behaviors of birds, ants, or bees, etc., BSO is inspired by the behavior of human beings, the most intelligent species on earth [9]. BSO embeds the constriction through clustering operation

Manuscript received January 29, 2013; revised March 26, 2013, May 05, 2013; accepted May 06, 2013. Date of publication May 13, 2013; date of current version September 20, 2013. Corresponding author: H. Duan (e-mail: hbduan@buaa.edu.cn).

Color versions of one or more of the figures in this paper are available online at http://ieeexplore.ieee.org.

Digital Object Identifier 10.1109/TMAG.2013.2262296 and expansion through individual generation into each generation of the algorithm, which may cause the BSO to have better balance between its exploration and exploitation search capability. Further discussions and modifications are conducted by Shi [10] and Zhan [11], confirming the feasibility and competence of BSO in function optimization problems.

In this paper, the novel thoughts of BSO are introduced to applied electromagnetic field to optimize design parameters of a DC brushless motor [12], [13] to maximize its efficiency. A predator-prey operation [14] is employed to reinforce the population diversity, as well as the extent of utilizing elite individuals and global information of the swarm.

The rest part of this paper is organized as follows. Section II demonstrates the principles of BSO and predator-prey operation. The optimization problem of a DC brushless motor is presented in Section III. Then in Section IV, experiments are carried out to compare the proposed PPBSO with the original BSO and PSO [13], [15]. Our concluding remarks are contained in Section V.

\section{Predator-Prey Based BSO}

\section{A. Brain Storm Optimization (BSO)}

As presented in [9] and [10], the process of BSO can be described as follows.

First, $m$ ideas are randomly initialized within the searching space, denoted as $X_{i}=\left[x_{i 1}, x_{i 2}, \ldots, x_{i D}\right]$, where $i=1,2, \ldots, m$, and $D$ is the dimension of the optimization problem to be solved. Each dimension signifies one design variable. Then each idea is evaluated and its fitness value $f\left(X_{i}\right)$ is obtained. The process of iteration begins afterwards.

During each generation, all the ideas are grouped into $k$ clusters using $k$-means clustering method, and the best idea in each cluster is chosen as the cluster center. Then a random center is replaced by a newly-generated idea with a probability of $p_{6 a}$, which is named as the replacing operation and has similar effect to the partial re-initialization operation in PSO and scouting operation in $\mathrm{ABC}[16]$. 
In the creating operation [11], BSO first randomly choose one cluster or two. Then the cluster center, which has higher priority or another idea in the cluster, is selected. After that, the selected idea(s) is updated according to (1):

$$
\begin{aligned}
x_{\text {new }} & =x_{\text {old }}+\xi N(\mu, \sigma) \\
x_{\text {old }} & = \begin{cases}x_{i j}, & \text { one cluster } \\
w_{1} x_{i 1, j}+w_{2} x_{i 2, j}, & \text { two clusters }\end{cases}
\end{aligned}
$$

where $N(\mu, \sigma)$ is the Gaussian random value with mean $\mu$ and variance $\sigma . w_{1}$ and $w_{2}$ are weight values of the two ideas. $\xi$ is an adjusting factor slowing the convergence speed down as the evolution goes, which can be expressed as

$$
\xi=r \operatorname{logsig}\left(\frac{N c_{\max } / 2-N c}{K}\right)
$$

where $r$ is a random value between 0 and $1 . N c_{\max }$ and $N c$ denote the maximum number of iteration and current number of iteration respectively. Whereas $K$ adjusts the slope of the logsig function. Such form of $\xi$ facilitates global searching ability at the beginning of the evolution and enhances local searching ability when the process is approaching to the end.

After the new idea is created, a crossover between the new one and the old one is conducted. The two ideas generated by crossover, together with the old one and the created one, are evaluated and the old one is replaced with the best of the four.

The process above repeats until $m$ ideas are updated. Thus, one generation is finished. The iteration goes until terminal requirement is met. Then the best idea is output as the optimal solution to the problem.

\section{B. Predator-Prey BSO (PPBSO)}

Taking a deep sight into the process of BSO described above, generally cluster centers are selected with higher probability than other ideas in the creating and updating section, therefore the cluster centers are treated with higher priority. Such mechanism emphasized the key role of the elite individuals in the swarm, but there seems to be some lack of directionality in it. In other words, global information of the entire swarm is not fully utilized. As for the diversity maintenance, re-initialization and stochastic search in the neighborhood are the main operations employed in BSO. Both of the two are frequently used in evolutionary algorithms.

In this paper, to better utilize the global information and further improve the evolutionary diversity of BSO, the concept of predator-prey is introduced [14], which is then used to improve the performance of BSO.

In our proposed PPBSO, the cluster centers act as the predators, having a trend moving towards better and better positions. Whereas the other ideas are preys, escaping from the nearest predator. As a result, the cluster centers can keep the best individuals of the swarm going towards the global best position, while the prey operation prevents the swarm from trapping in local optimum. In PPBSO, (1) is transformed to (4) and (5):

$$
\begin{aligned}
x_{\text {pred }}= & x_{\text {old }}+\xi N(\mu, \sigma)+w_{\text {pred }}\left(x_{\text {gbest }}-x_{\text {old }}\right) \\
x_{\text {prey }}= & x_{\text {old }}+\xi N(\mu, \sigma) \\
& -P a \operatorname{sgn}\left(x_{\text {center }}-x_{\text {old }}\right) e^{-b\left|x_{\text {center }}-x_{\text {old }}\right|}
\end{aligned}
$$

\begin{tabular}{|c|c|c|c|}
\hline & Variables & Description & Value \\
\hline $\begin{array}{l}\text { Objective } \\
\text { variable }\end{array}$ & $\eta(\%)$ & Efficiency & - \\
\hline \multirow{5}{*}{$\begin{array}{c}\text { Design } \\
\text { variables }\end{array}$} & $D_{s}(\mathrm{~mm})$ & Stator diameter & {$[150,330]$} \\
\hline & $B_{d}(\mathrm{~T})$ & $\begin{array}{l}\text { Mean magnetic flux } \\
\text { density in the teeth }\end{array}$ & {$[0.9,1.8]$} \\
\hline & $\delta\left(\mathrm{A} / \mathrm{mm}^{2}\right)$ & $\begin{array}{l}\text { Density current on } \\
\text { the windings }\end{array}$ & {$[2.0,5.0]$} \\
\hline & $B_{e}(\mathrm{~T})$ & $\begin{array}{c}\text { Magnetic flux } \\
\text { density in the air gap }\end{array}$ & {$[0.5,0.76]$} \\
\hline & $B_{c s}(\mathrm{~T})$ & $\begin{array}{c}\text { Mean magnetic flux } \\
\text { density in the stator } \\
\text { back iron }\end{array}$ & {$[0.6,1.6]$} \\
\hline \multirow{6}{*}{$\begin{array}{c}\text { Fixed } \\
\text { variables }\end{array}$} & $L_{m}(\mathrm{~mm})$ & $\begin{array}{l}\text { Magnetic length of } \\
\text { the motor }\end{array}$ & 45 \\
\hline & $r_{r s}(\mathrm{~mm})$ & $\begin{array}{l}\text { Ratio of the length } \\
\text { rotor-stator }\end{array}$ & 1.11 \\
\hline & $e(m m)$ & Air gap & 0.8 \\
\hline & $U_{D C}(\mathrm{~V})$ & DC bus voltage & 120 \\
\hline & $B_{c r}(\mathrm{~T})$ & $\begin{array}{l}\text { Mean magnetic flux } \\
\text { density in the rotor } \\
\text { back iron }\end{array}$ & 1.2 \\
\hline & $p$ & $\begin{array}{l}\text { Number of } \\
\text { pole-pairs }\end{array}$ & 6 \\
\hline
\end{tabular}

TABLE I

OPTIMIZATION PARAMETERS

TABLE II

CONSTRAINTS

\begin{tabular}{ccc}
\hline Variables & Description & Value \\
\hline$M_{\text {tot }}(\mathrm{kg})$ & $\begin{array}{c}\text { Total mass of the active } \\
\text { parts }\end{array}$ & $M_{t o t} \leq 15$ \\
\hline$D_{\text {ext }}(\mathrm{mm})$ & outer diameter & $D_{e x t} \leq 340$ \\
\hline$D_{\text {int }}(\mathrm{mm})$ & inner diameter & $D_{i n t \geq 76}$ \\
\hline$I_{\max }(\mathrm{A})$ & $\begin{array}{c}\text { Maximum current in the } \\
\text { phases }\end{array}$ & $I_{\max } \geq 125$ \\
\hline $\operatorname{discr}\left(D_{s}, \delta, B_{d}, B_{e}\right)$ & $\begin{array}{c}\text { Determinant used for the } \\
\text { calculation of the slot } \\
\text { height }\end{array}$ & $\operatorname{discr}\left(D_{s}, \delta, B_{d}, B_{e}\right) \geq 0$ \\
\hline$\left.T_{a}{ }^{\circ} \mathrm{C}\right)$ & temperature of the motor & $T_{a} \leq 120$ \\
\hline
\end{tabular}

where $x_{\text {pred }}, x_{\text {prey }}$, and $x_{\text {center }}$ denote new position of predator, new position of prey and the nearest predator to the current prey respectively. $P$ is a binary variable determining whether a prey escapes or not. $a$ and $b$ are two factors measuring the difficulty of escaping. Specifically

$$
a=x_{\mathrm{span}}, b=\frac{100}{x_{\mathrm{span}}}
$$

where $x_{\mathrm{span}}$ is the searching range of the design variable.

The detailed procedure of PPBSO is presented as follows:

Step 1: Initialization. Number of ideas and maximum number of iterations are respectively assigned as $m$ and $N c_{\text {max }} \cdot m$ ideas are randomly generated.

Step 2: Clustering.

Step 3: With probability $p_{5 a}$, replace a randomly selected cluster center with a randomly generated idea.

Step 4: With probability $p_{6 b}$, pick one cluster, otherwise, pick two clusters.

Step 5: With probability $p_{6 b 3}$, pick cluster center(s) and go to Step 6, otherwise pick other ideas and go to Step 7.

Step 6: Conduct the predator operation and generate new idea according to the best idea. Then go to Step 8 . 
TABLE III

CONTROL PARAMETERS OF BSO AND PPBSO

\begin{tabular}{c|c|c}
\hline Parameter & Description & Value \\
\hline$m$ & Number of ideas & 30 \\
\hline$N c_{\max }$ & Maximum times of iteration & 200 \\
\hline$K$ & Number of clusters & 3 \\
\hline$p_{5 a}$ & $\begin{array}{c}\text { Probability to directly update a cluster } \\
\text { center }\end{array}$ & 0.2 \\
\hline$p_{6 b}$ & Probability to choose one cluster & 0.8 \\
\hline$p_{6 b 3}$ & $\begin{array}{c}\text { Probability to select the center of the } \\
\text { selected cluster }\end{array}$ & 0.4 \\
\hline$p_{6 c}$ & $\begin{array}{c}\text { Probability to select the centers of the } \\
\text { two selected clusters }\end{array}$ & 0.5 \\
\hline$w_{\text {predator }}$ & Weight factor of the predator operator & 0.05 \\
\hline$p_{\text {prey }}$ & Probability to conduct prey operator & 0.1 \\
\hline \multicolumn{2}{|c}{}
\end{tabular}

TABLE IV

CONTROL PARAMETERS OF PSO

\begin{tabular}{c|c|c}
\hline Parameter & Description & Value \\
\hline$m$ & Number of particles & 30 \\
\hline$N c_{\max }$ & Maximum times of iteration & 1000 \\
\hline$c_{1}$ & Inertia factor & {$[0.4,1]$} \\
\hline$c_{2}$ & Self best factor & 1.6 \\
\hline$c_{3}$ & Global best factor & 1.8 \\
\hline
\end{tabular}

TABLE V

SIMULATION RESULTS

\begin{tabular}{c|c|c|c|c}
\hline \multirow{2}{*}{ Methods } & \multicolumn{2}{|c|}{ Efficiency } & \multicolumn{2}{c}{ CPU time(s) } \\
\cline { 2 - 5 } & average & $\begin{array}{c}\text { standard } \\
\text { deviation }\end{array}$ & average & $\begin{array}{c}\text { standard } \\
\text { deviation }\end{array}$ \\
\hline BSO & $95.31 \%$ & $7 \times 10^{-5}$ & 3.92 & 0.13 \\
\hline PPBSO & $95.32 \%$ & $4.9 \times 10^{-5}$ & 3.98 & 0.11 \\
\hline PSO & $94.94 \%$ & $4.7 \times 10^{-3}$ & 3.06 & 0.93 \\
\hline
\end{tabular}

TABLE VI

BEST SOLUTIONS OF BSO, PPBSO, AND PSO

\begin{tabular}{cccccccc}
\hline & $\eta(\%)$ & $\begin{array}{c}D_{s} \\
(\mathrm{~mm})\end{array}$ & $\begin{array}{c}B_{d} \\
(\mathrm{~T})\end{array}$ & $\begin{array}{c}\delta \\
\left(\mathrm{A} / \mathrm{mm}^{2}\right)\end{array}$ & $B_{e}(\mathrm{~T})$ & $B_{c s}(\mathrm{~T})$ & $\begin{array}{c}\text { CPU } \\
\text { time } \\
(\mathrm{s})\end{array}$ \\
\hline BSO & 95.32 & 201.3 & 1.8 & 2.070 & 0.648 & 0.876 & 3.73 \\
\hline $\begin{array}{c}\text { PP } \\
\text { BSO }\end{array}$ & 95.32 & 201.3 & 1.8 & 2.057 & 0.649 & 0.884 & 3.71 \\
\hline PSO & 95.18 & 150.0 & 1.8 & 2.0 & 0.572 & 1.6 & 2.91 \\
\hline $\begin{array}{c}\text { Opti- } \\
\text { mal }\end{array}$ & 95.32 & 201.2 & 1.8 & 2.044 & 0.648 & 0.896 & - \\
\hline
\end{tabular}

Step 7: Conduct the prey operation and generate new idea with tendency of moving away from the nearest cluster center.

Step 8: Conduct crossover operation and select the best idea among the group consisting of the old idea, the newly generated idea and the two ideas produced by the crossover operation. Replace the old idea with the selected one.

Step 9: If $m$ ideas have been updated, go to Step 10. Otherwise go back to Step 4 .

Step 10: Evaluate the $m$ ideas.

Step 11: If the current number of iterations $N c$ is less than $N c_{\text {max }}$, go back to Step 2. Otherwise the algorithm is terminated and the best idea is output as the solution to the optimization problem.

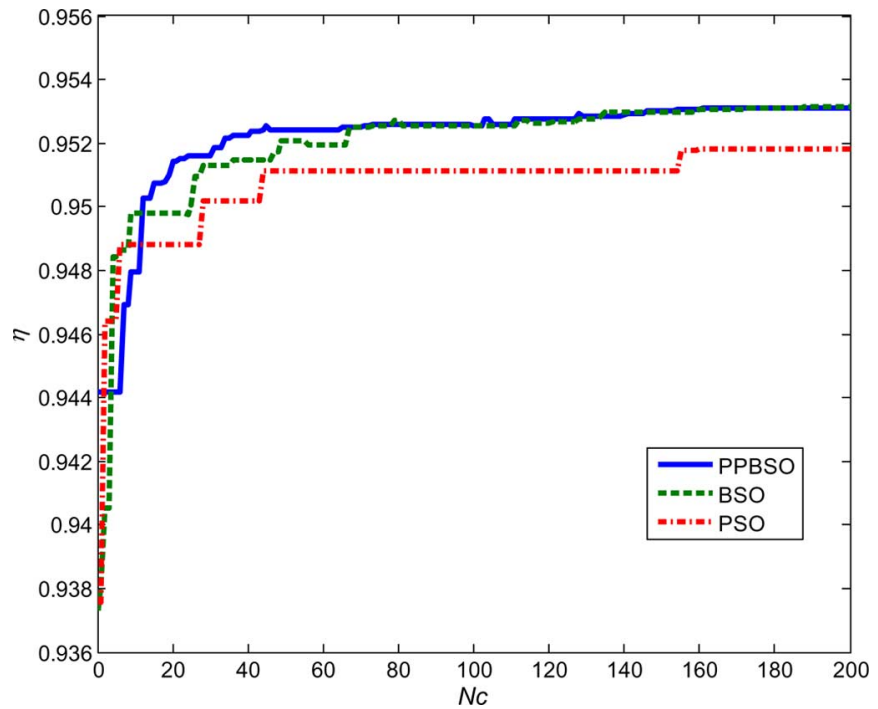

Fig. 1. Evolutionary process comparison of PPBSO, BSO, and PSO $(N c=$ 200)

The flow chart of the optimization is presented in Fig. 2.

\section{PROBlem Formulation}

In order to analyze the feasibility of BSO and the improved PPBSO method, the optimization for an analytical model of a brushless DC wheel motor is chosen as the benchmark [12]. The model of the brushless DC wheel motor and three affiliated optimization benchmarks were proposed in [12]. In this work, the first benchmark of the three proposed ones, namely the five parameters problem, is discussed and optimized. As is shown in Table I, this benchmark problem has five design variables and one efficiency value. The efficiency is determined by these variables, and our object is to maximize the efficiency. The constraints are shown in Table II. The optimization is aimed at obtaining the best efficiency within the allowable parameter range.

The five parameter problem can be expressed as the following general optimization problem:

$$
\begin{aligned}
& \operatorname{maximize} \eta=f\left(D_{s}, B_{d}, \delta, B_{e}, B_{\mathrm{cs}}\right) \\
& \text { with } \\
& 150 \leq D_{s} \leq 330,0.9 \leq B_{d} \leq 1.8,2.0 \leq \delta \leq 5.0, \\
& 0.5 \leq B_{e} \leq 0.76,0.6 \leq B_{c s} \leq 1.6 \\
& \text { s.t. } \\
& M_{\mathrm{tot}} \leq 15, D_{\text {ext }} \leq 340, D_{\text {int }} \geq 76, \\
& I_{\max } \geq 125, \operatorname{discr}\left(D_{s}, B_{d}, \delta, B_{e}\right) \geq 0, T_{a} \leq 120 .
\end{aligned}
$$

\section{OPTIMIZATION RESUltS}

For comparison purpose, PSO [13], BSO and PPBSO are applied to optimize the brushless DC wheel motor. The control parameters of BSO and PPBSO are given in Table III. The parameters for PSO are adopted from [13] and are shown in Table IV. The population size is set equal to those of BSO and PPBSO. Because of the operations in Step 8 and Step 10 in Section II, the efficiency is calculated 5 times in the process of updating one individual. Therefore the maximum generation of PSO is 


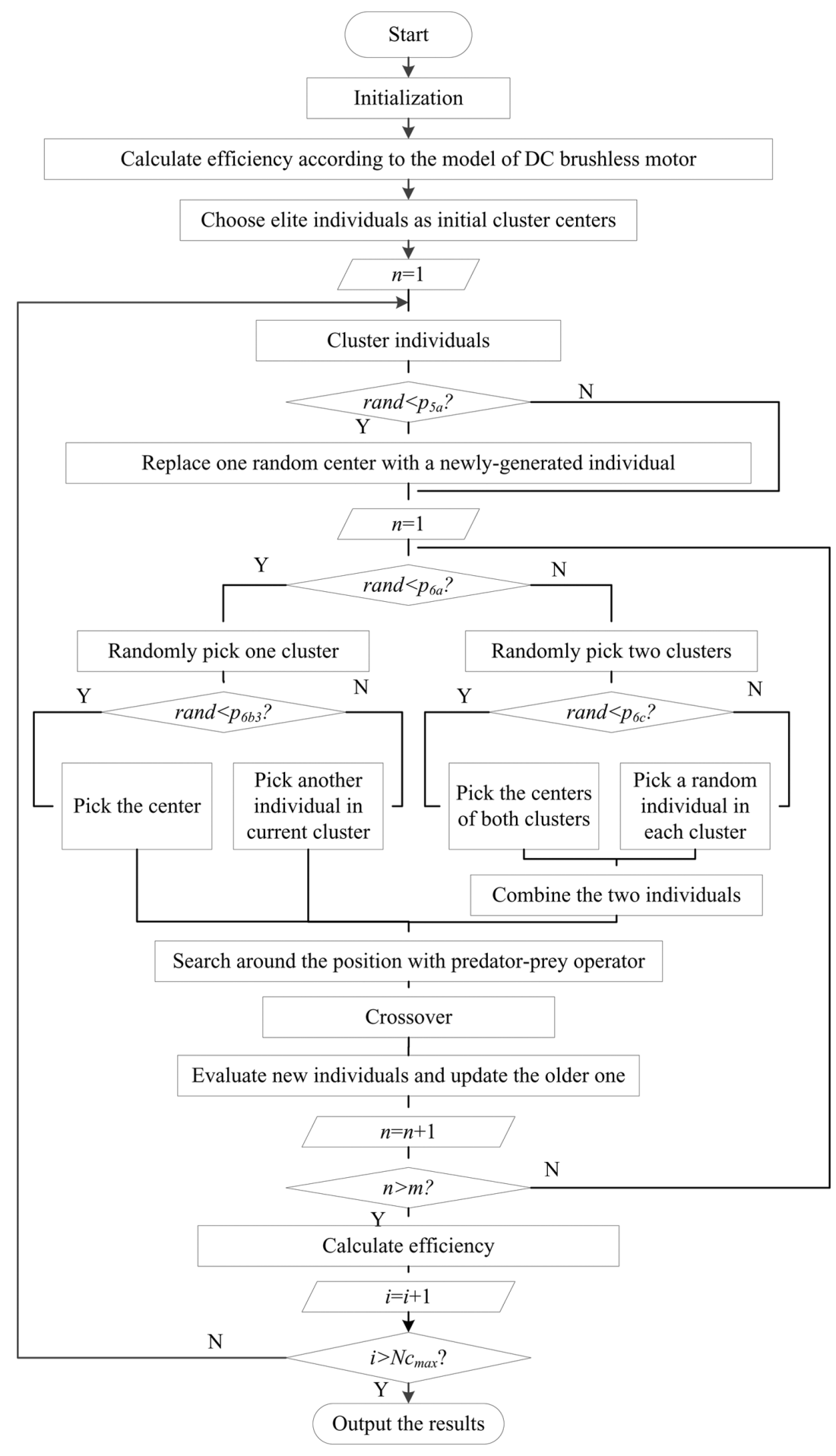

Fig. 2. The detailed flow chart of PPBSO.

set to 5 times of BSO's to let them have the same amount of calculation.

Since the constraints in Table II should be strictly met in the design of the motor, the individuals which do not satisfy the constraints are abandoned. Specifically, the efficiency values of these individuals are set to 0 .

The statistic result of the simulations is presented in Table V. Table VI shows the best solutions among the 20 trials of the three algorithms.
The last row in Table VI presents the theoretic optimum given in [12] and [17]. From Tables V and VI, we can conclude that both BSO and PPBSO can obtain an appreciable approximation of the global optimum, yet the average value and stability of PPBSO is slightly better than the original one. Though most parameters in PSO are the same as that in [13], the simulation result of PSO did not turn out to be as good as the established results: PSO is very likely to trap in local optima and the average value given by PSO is overwhelmed by those of BSO and 
PPBSO. Among the three, PPBSO has the lowest standard deviation value of its solutions. In fact, it always reaches a solution greater than $95.31 \%$ in the 20 trials, while the best result of PSO is only $95.18 \%$. For a swarm intelligence algorithm, it is not likely to precisely find the optimal solution, and each time the solutions may be different. As a result, PPBSO, who can reliably find a solutions close enough to optimum, is a feasible approach to the motor optimization problem.

To directly illustrate the performance during the evolutionary process of the proposed PPBSO, the best results obtained by the three methods after 200 generations of evolution are displayed in Fig. 1.

Owing to the predator-prey operator, PPBSO exhibits better capability to jump out of local optimum. When the individuals converge to a local optimum, indicated by the horizontal parts of the efficiency curve, PPBSO takes much less generations to jump out of the local optimum and find a position with higher efficiency. Therefore, the curve of PPBSO is much smoother, and is always above those of BSO and PSO except at the very beginning.

\section{CONCLUSION}

BSO is a newly-proposed swarm intelligence optimization algorithm inspired by brain storming behavior of human being. Positions in the searching space can be denoted as different ideas. In each generation of the evolution, the operations of clustering, replacing and updating are conducted. Ideas are updated by searching in their neighborhoods and combining with ideas from other clusters. BSO is already proven competent in function optimization problems.

This paper modified the original BSO with the operators of predator-prey to better utilize the global information and enhanced the swarm diversity during evolution to avoid local optima. The proposed PPBSO was successfully applied to the field of electromagnetics to optimize the design variables of a DC brushless motor. Experimental results on optimizing the motor variables validated the feasibility of BSO and PPBSO in comparison with PSO. What is more, PPBSO exhibited better ability to jump out of local optimum and shows satisfactory stability in repeated experiments, which is especially meaningful as a swarm intelligence algorithm.

\section{ACKNOWLEDGMENT}

This work was supported by Natural Science Foundation of China (NSFC) under Grant \#61333004, \#61273054, \#61273367, \#60975072, and \#60975080, National Key Basic Research Program of China (973 Program) under Grant \#2014CB046401 and \#2013CB035503, Open Fund of the
State Key Laboratory of Virtual Reality Technology and Systems under Grant \#VR-2013-ZZ-02, Program for New Century Excellent Talents in University of China under Grant \#NCET-10-0021, Top-Notch Young Talents Program of China, Graduate Innovation Foundation for Beihang University under Grant \#YCSJ-01-201206, and Aeronautical Foundation of China under Grant \#20115151019.

\section{REFERENCES}

[1] J. Kennedy, R. C. Eberhart, and Y. H. Shi, Swarm Intelligence. San Mateo, CA: Morgan Kaufmann, 2001.

[2] B. Sareni, L. Krähenbühl, and A. Nicolas, "Efficient genetic algorithms for solving hard constrained optimization problems," IEEE Trans. Magn., vol. 36, no. 4, pp. 1027-1030, Jul. 2000.

[3] C. H. Im, H. K. Jung, and Y. J. Kim, "Hybrid genetic algorithm for electromagnetic topology optimization," IEEE Trans. Magn., vol. 39, no. 5, pp. 2163-2169, Sep. 2003.

[4] G. Crevecoeur, P. Sergeant, L. Dupré, and R. Van de Walle, "A twolevel genetic algorithm for electromagnetic optimization," IEEE Trans. Magn., vol. 46, no. 7, pp. 2585-2595, Jul. 2010.

[5] X. Meng, S. Wang, J. Qiu, J. Zhu, Y. Wang, Y. Guo, D. Liu, and W. $\mathrm{Xu}$, "Dynamic multilevel optimization of machine design and control parameters based on correlation analysis," IEEE Trans. Magn., vol. 46, no. 8, pp. 2779-2782, Aug. 2010.

[6] U. Baumgartner, Ch. Magele, and W. Renhart, "Pareto optimality and particle swarm optimization," IEEE Trans. Magn., vol. 40, no. 2, pp. 1172-1175, Mar. 2004.

[7] L. S. Coelho and P. Alotto, "Gaussian artificial bee colony algorithm approach applied to Loney's solenoid benchmark problem," IEEE Trans. Magn., vol. 47, no. 5, pp. 1326-1329, May 2011.

[8] M. C. Silva, L. S. Coelho, and L. Lebensztajn, "Multiobjective biogeography-based optimization based on predator-prey approach," IEEE Trans. Magn., vol. 48, no. 2, pp. 951-954, Feb. 2012.

[9] Y. Shi, "Brain storm optimization algorithm," in Proc. 2nd Int. Conf. Swarm Intelligence, Chongqing, China, Jun. 12-15, 2011, pp. 303-309.

[10] Y. Shi, "An optimization algorithm based on brainstorming process," Int. J. Swarm Intell. Res., vol. 2, no. 4, pp. 35-62, Oct.-Dec. 2011.

[11] Z. Zhan, J. Zhang, Y. Shi, and H. Liu, "A modified brain storm optimization," in Proc. 2012 IEEE World Congr. Computational Intelligence, Brisbane, Australia, Jun. 10-15, 2012, pp. 1-8.

[12] S. Brisset and P. Brochet, "Analytical model for the optimal design of a brushless DC wheel motor," COMPEL: Int. J. Comput. Math. Elect. Electron. Eng., vol. 24, no. 3, pp. 829-848, 2005.

[13] F. Moussouni, S. Brisset, and P. Brochet, "Comparison of two multiagent algorithms: ACO and PSO for optimization of the brushless DC motor," Studies Comput. Intell., vol. 119, no. 3, pp. 3-10, 2008.

[14] M. Higashitani, A. Ishigame, and K. Yasuda, "Particle swarm optimization considering the concept of predator-prey behavior," in Proc. IEEE Congr. Evolutionary Computation, Vancouver, BC, Canada, Jul. 16-21, 2006, pp. 434-437.

[15] Y. Shi and R. Eberhart, "A modified particle swarm optimizer," in Proc. IEEE Int. Conf. Evolutionary Computation, Anchorage, AK, May 4-9, 1998, pp. 69-73.

[16] C. F. Xu, H. B. Duan, and F. Liu, "Chaotic artificial bee colony approach to uninhabited combat air vehicle (UCAV) path planning," Aerospace Sci. Technol., vol. 14, no. 8, pp. 535-541, Dec. 2010.

[17] F. Moussouni and S. Brisset, "A benchmark for a mono and multi objective optimization of the brushless DC wheel motor," Jul. 2008 [Online]. Available: http://12ep.univ-lille1.fr/come/benchmark-wheelmotor.htm 